\title{
Automatic Color Detection of Archaeological Pottery with Munsell System
}

\author{
Filippo Stanco ${ }^{1}$, Davide Tanasi ${ }^{2}$, Arcangelo Bruna ${ }^{1}$, and Valentina Maugeri \\ ${ }^{1}$ Dipartimento di Matematica e Informatica, Università di Catania, \\ viale A. Doria, 6 - 95125 Catania, Italy \\ \{fstanco, bruna\}@dmi. unict. it \\ ${ }^{2}$ Arcadia University, Mediterranean Center for Arts and Sciences, \\ Palazzo Ardizzone, via Roma, 124 - 96100 Siracusa, Italy \\ dtanasi@mediterranencenter.it
}

\begin{abstract}
A main issue in the archaeological research is the identification of colored surfaces and soils through the application of Munsell system. This method widely used also in other fields, like geology and anthropology, is based on the subjective matching between the real color and its standardized version on Munsell chart. For preventing many possible errors caused by the subjectivity of the system itself, in this paper an automatic method of color detection on selected regions of digital images of archaeological pottery is presented.
\end{abstract}

Keywords: Archeological artifacts, color matching, Munsell color space.

\section{Introduction}

"What is color? It's a sensation, like hunger or fatigue, that exists only in our minds. Like hunger and fatigue, it's caused by external factors that we can measure and quantify, but measuring those external factors is no more measuring color than counting calorie intake tells us how hungry we are, or measuring exercise tells us how fatigued" [4].

Every observer perceives color differently. A major obstacle encountered when comparing colors is the choice of descriptive words. Color also varies in its appearance due to changes in the light source and the distance of the light source. The color identification as any other cognitive process can also be seriously influenced by cultural and linguistic background as well as psychological state [2]. Furthermore, it must also be taken into account that colors can only be described unequivocally as long as all the interlocutors can actually see them. If, however, one scholar receives the information exclusively from the oral or written reports of one of the others, she or he must try to picture a particular color without having perceived it herself or himself. The mental image thus created will thereby only in the rarest cases correspond to the visual impression which the other person was stimulated to communicate. 
Since color can only inadequately be described by verbal means, nowadays whenever one wants to make unequivocal systematically, constructed color chart are used.

At the beginning of the 20th century, Albert H. Munsell [5] brought clarity to color communication by establishing an orderly system for accurately identifying every color that exists. The Munsell color system is a way of precisely specifying colors and showing the relationship among colors. Every color has three qualities or attributes: hue, value and chroma. Munsell established numerical scales with visually uniform steps for each of these attributes.

Hue is that attribute of a color by which we distinguish red from green, blue from yellow, and so on. There is a natural order of hues: red, yellow, green, blue, purple. Then five intermediate hues were inserted: yellow-red, green-yellow, blue-green, purple-blue and red-purple, making ten hues in all. Paints of adjacent colors in this series can be mixed to obtain a continuous variation from one color to the other. For simplicity, the initials as symbols to designate the ten hue sectors are used: R, YR, Y, GY, G, BG, B, PB, P and RP.

Value indicates the lightness of a color. The scale of value ranges from 0 for pure black to 10 for pure white. Black, white and the grays between them are called "neutral colors", because they have no hue like the other "chromatic colors", that have it.

Chroma is the degree of departure of a color from the neutral color of the same value. The scale starts at zero, for neutral colors, but there is no arbitrary end to the scale, as new pigments gradually become available.

The Munsell color-order system has gained international acceptance. It is recognized in standard Z138.2 of the American National Standards Institute; Japanese Industrial Standard for Color, JIS Z 872; the German Standard Color System, DIN 6164; and several British national standards.

The reliability of Munsell's color scheme has been recently stressed by specific neurobiological researches which demonstrated how that system has successfully standardized color in order to match the reflectance spectra of Munsell's color chips with the sensitivity of the cells in the lateral geniculate nucleus (LGN cells), responsible for color identification. This statement makes Munsell charts appropriate for almost all jobs that require manual color identification by human agent [3].

In archaeology Munsell charts are widely used as the standard for color identification of soil profiles, organic materials, rock materials, colored glasses, metals, paintings, textiles and mainly pottery.

For which regards the interpretation of pottery the precise color identification of such parts like clay body, treated surfaces, core, and outer layers like slip and painting, it is fundamental for defining its stylistic and technical features.

As a coding framework, the charts both mediate perceptual access to the colored object being classified, and provide a color reference standard. This tool does not stand alone as a self-explicating artifact; instead its proper use is embedded within a set of systematic work practices, varying from community to community. As demonstrated in application in fields of archaeology, anthropology, these practices can contribute to misclassification of colors [2]. In fact, Munsell notations are not always unequivocal and the limits of their use are well known since decades [1]. 
Besides the above mentioned cultural, linguistic and psychological background, several other factors can misled the observer in the task of color identification of pottery. The most common are surface homogeneity of the material, state of color surface, color type, test condition, accuracy of assertion, color blindness, quality and type of the Munsell charts.

While Munsell system is ideally shaped for smoothed surfaces no displaying disturbing textures, the pottery surfaces are just in rarest cases homogenous both in relation in their color and their texture, often altered by cracks and superficial voids. Decorative techniques aimed to smooth, coat or glaze can also modify the real chromatic value of the surface. Some kind of patina and incrustation can cause misinterpretation of the color as well as artificial light sources, different than natural daylight must be avoided. Finally, an additional human error can be determined by the inaccuracy caused by tasks involving thousands of checks and by problems coming from quite common deficiencies in color perception [1]. In this perspective, the development of an automatic system for classification of colors in archaeology, and in particular in the field of pottery research, must be considered crucial for providing a solution to all the above mentioned problems.

In this paper, we propose an algorithm to extract an objective Munsell definition of colored selected regions of digital image. The method corrects the illumination defects in the picture in order to create the ideal illumination that permits one to extract the color information.

The rest of the paper is organized as follows. In Section 2 the proposed technique is described; the next Section reports a series of experiments devoted to assess the effectiveness of the method. Finally, some conclusions are given together with a few hints for the future work.

\section{Proposed System}

The proposed system is a semi-automatic algorithm aiming to find the best match between a user selected color in an archeological sherd with a color in the Munsell charts [5]. Focusing on a particular color in the sherd, the system must provide the color in the Munsell table that best matches it. There are several problems to overcome: first of all, the acquisition process is not usually obtained in good illumination conditions. Pictures are often acquired in artificially illuminated rooms, with uncontrolled light sources. It means that the color correction of the camera is not always able to compensate correctly for the illuminant. This problem, known as "white balance", is a main issue to deal with [6]. Secondly, the patch that the user is asked to point should be representative of the region. Noises (especially for low illuminated acquisitions) and dirty spots can make really difficult this process. Lastly, also the matching is not a minor problem, since the colors cannot be simply represented in the Munsell table; hence a different data space must be used. We define a system and a pipeline to overcome all these problems. A database has also been set up to make the tests and it is available in [7] to let the reader use and/or extend it for further research. 
The proposed pipeline (Figure 1) is composed by a color correction module, a patch extraction, and a color matching. In the next sub-sections each block is analyzed in detail.

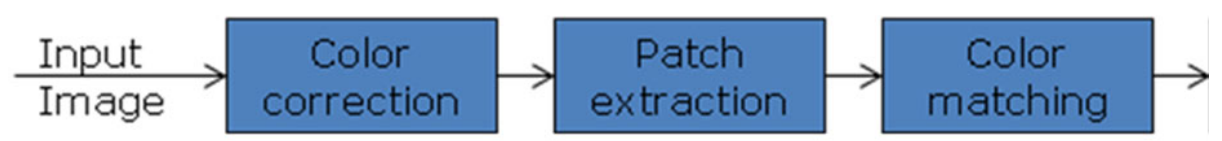

Fig. 1. Block scheme of the proposed algorithm pipeline

\subsection{Color Correction}

In the color correction module, the image is compensated for the illuminant. This problem is known as "white balance" and there are lots of algorithms in literature to reduce the problem in a fully automatic way $[6,8,9,10]$. Unfortunately, a zero failure algorithm does not exist, since the white balance is an ill-posed problem and all the methods available are based on assumptions. Whenever, when the assumptions are not verified, the algorithm fails [11]. Moreover there is another problem: the pictures are obtained from a camera and the white balance is already applied (like other algorithms, e.g., color matrix, gamma correction, etc.). It may produce problems in color reproduction. In order to control these problems, we started taking pictures with a color checker chart acquired in the same image: first to obtain the best correction (to validate all the other steps of the algorithm); and second to create a ground truth to validate further methodologies.

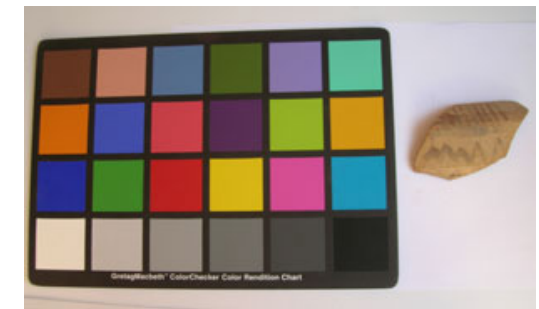

(a)

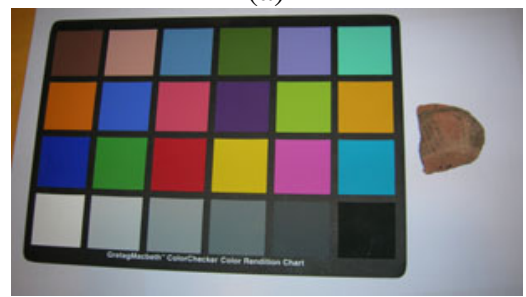

(c)

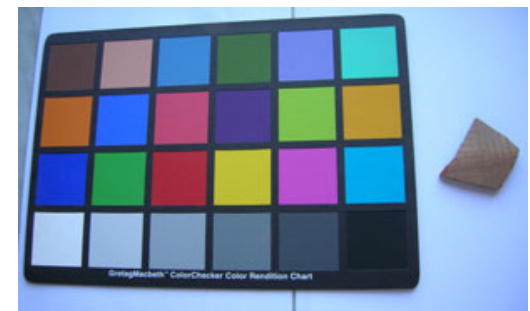

(b)

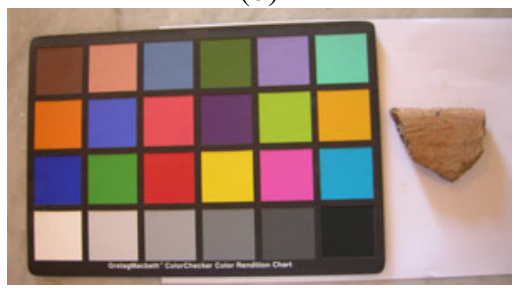

(d)

Fig. 2. Examples from the dataset with different illuminants; the effect of the illumination conditions is evident 

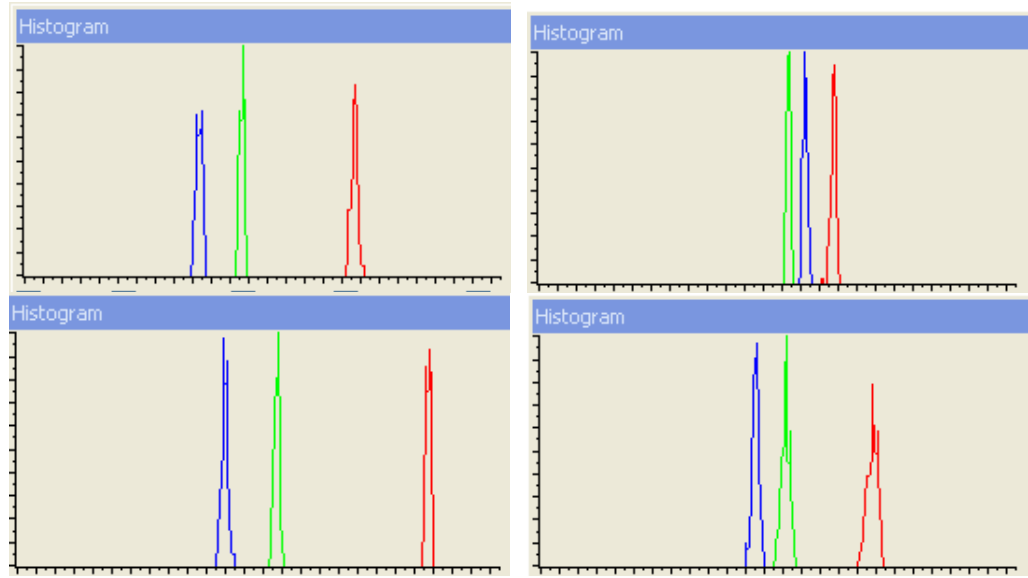

Fig. 3. Histogram of the Macbeth chart 'light skin' patch in the four images in Figure 2. The RGB mean values are, respectively (from top to bottom, from left to right): (177-116-93), $(157,143,133),(217,135,110),(181,133,116)$.

In the Figure 2 some pictures of the dataset are shown. They were acquired in different illumination conditions. In Figure 3 the related histograms of the 'light skin' patch (the second patch of the Macbeth chart) are shown. It is evident the effect of the illuminant on the color rendition: without any post-processing correction, the color matching is impossible.

The algorithm proposed supposes that images are acquired with the Macbeth chart and the correction is performed compensating some patches of the chart.

It is supposed to compensate for the illuminant according to the von Kries-Ives adaptation [12], i.e., the correction can be obtained by multiplying every color component with an amplification coefficient:

$$
\left[\begin{array}{l}
R_{\text {out }} \\
G_{\text {out }} \\
B_{\text {out }}
\end{array}\right]=\left[\begin{array}{ccc}
g_{r} & 0 & 0 \\
0 & g_{g} & 0 \\
0 & 0 & g_{b}
\end{array}\right] \cdot\left[\begin{array}{l}
R_{\text {in }} \\
G_{\text {in }} \\
B_{\text {in }}
\end{array}\right]
$$

Where (Rin, Gin,Bin) is the original triplet, $\left(R_{\text {out }}, G_{\text {out }}, B_{\text {out }}\right)$ is the corrected value and $\left(g_{r}, g_{g}, g_{b}\right)$ are the gains.

The weights can be found in different ways. Taking into account the Macbeth chart, the weights could be retrieved by constraining one of the patch to be same (e.g., in the $s R G B$ color space) to the real value (since all the patches are completely characterized).

$$
\left[\begin{array}{l}
g_{r} \\
g_{g} \\
g_{b}
\end{array}\right]=\left[\begin{array}{l}
R_{t} / R_{i n} \\
G_{t} / G_{i n} \\
B_{t} / B_{i n}
\end{array}\right]
$$


Where $\left(R_{t}, G_{t}, B_{t}\right)$ is the target sRGB triplet of the used patch $(201,201,201)$ for the grey patch highlighted in the Figure 2.

A better way is to obtain the coefficients by using more patches. In this case a set of redundant equations are obtained, hence optimization techniques, e.g. Least Squares Method, can be used to obtain the gains. The error function to be minimized is the following:

$$
E=\sum_{i=1}^{p} e_{i}^{2}
$$

Where $E$ is the error function, $p$ is the number of selected patches; $e_{i}$ is the error contribution of each patch:

$$
e_{i}=\left|\left(g_{r} \cdot R_{i n}\right)-R_{t}\right|+\left|\left(g_{g} \cdot G_{i n}\right)-G_{t}\right|+\left|\left(g_{b} \cdot B_{i n}\right)-B_{t}\right|
$$

This formula provided good results in terms of visual quality. Other error measures can be used, e.g., in a more perceptually uniform color space.

In our system we started using the six gray patches in the bottom of the chart. Of course, in order to reduce the noise, the patch color is obtained as mean of a patch crop. The entire process is shown in Figures 4 and 5.

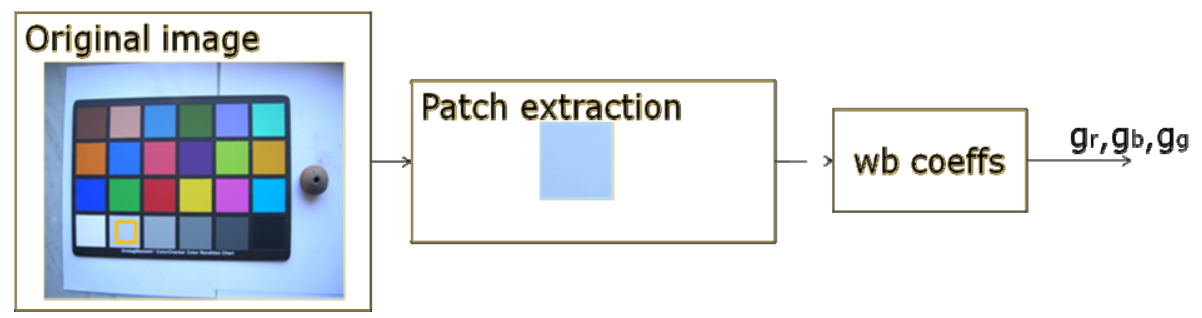

Fig. 4. Color correction module using one neutral patch

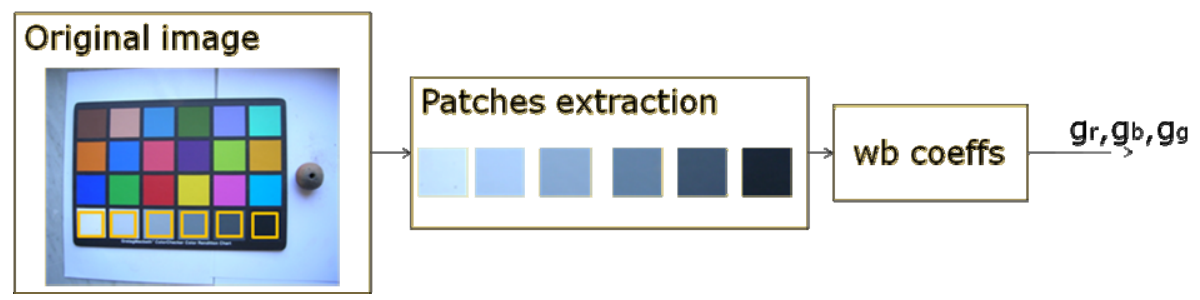

Fig. 5. Color correction module using six neutral patches

In the 'Patch extraction', user has to select the patches and the system retrieves the mean value of the patch. In the ' $w b$ coeffs' block the system computes the gains according to the formulas shown above. 


\subsection{Patch Extraction}

After the color correction, the user has also to choose the color to be matched in the Munsell table. A 'point and click' is the best user friendly way to do it. In order to reduce difficulties due to noises or scratches in the archeological finds, when the user points over a colored surface, a homogeneous patch is shown. The color of the patch is obtained, for the generic pointed pixel at position $(p, q)$, as median of a square window:

$$
C=\operatorname{median}\left(C_{i, j} / i, j=p-n, \ldots, p+n\right)
$$

Where $C=R, G$, or $B ; n=10$ in the actual implementation. The use of the median instead of the mean value allows reducing the influences of impulsive noises and scratches in the patch extraction. In the Figure 6 is shown a snapshot of the software.

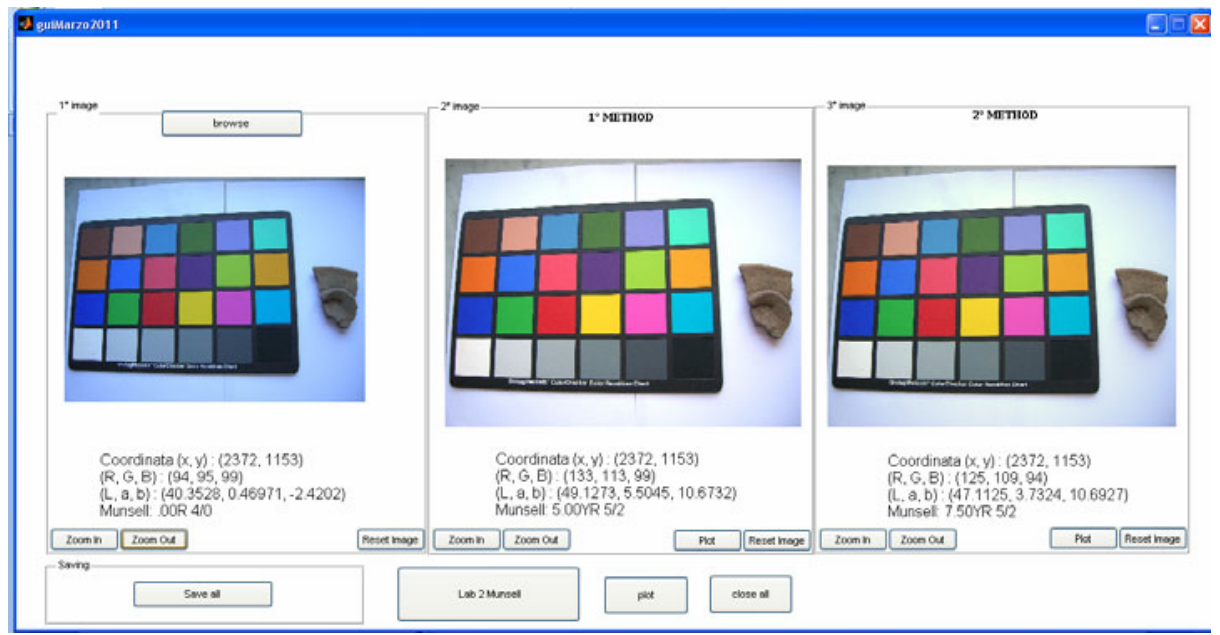

Fig. 6. A snapshot of our system

\subsection{Color Matching}

The color matching block aims to obtain the color in the Munsell table most similar to the patch chosen by the user. Each color is represented by three components: hue $(\mathrm{H})$, value (V) and chroma (C). The representation is "H V/C". As example, 10YR 6/4 means that the hue is $10 \mathrm{YR}$, i.e., a combination of yellow and red (note that the defined colors are $\mathrm{R}=\mathrm{Red}, \mathrm{Y}=$ Yellow, $\mathrm{G}=\mathrm{Green}, \mathrm{B}=\mathrm{Blue}$, and $\mathrm{P}=\mathrm{Purple}$ ); the value is 6 and the chroma is 4 .

The main problem is that there is no direct formula to convert from Munsell patches to a representation in other color spaces. It also means that there is no way to work directly in the Munsell space, since it is quite difficult to define a distance 
measure to find the best fit. The earliest Munsell based difference formulae is Nickerson's “index of fading” [13] defined as:

$$
\Delta E=\frac{2}{5} \mathrm{C} \Delta \mathrm{H}+6 \Delta \mathrm{V}+3 \Delta \mathrm{C}
$$

Where $\mathrm{H}, \mathrm{V}$, and $\mathrm{C}$ are Munsell coordinates.

It is a very old measure and authors decided to use also another measure using a perceptive uniform color representation. The DeltaE94 in the $L^{*} a * b *$ color space has been chosen. It is approximately perceptually uniform, i.e. a change of the same amount in a color value produces a change of about the same visual importance.

All the patches in the Munsell table were represented in the Lab color space. The block based scheme of the color matching phase is shown in Figure 7.

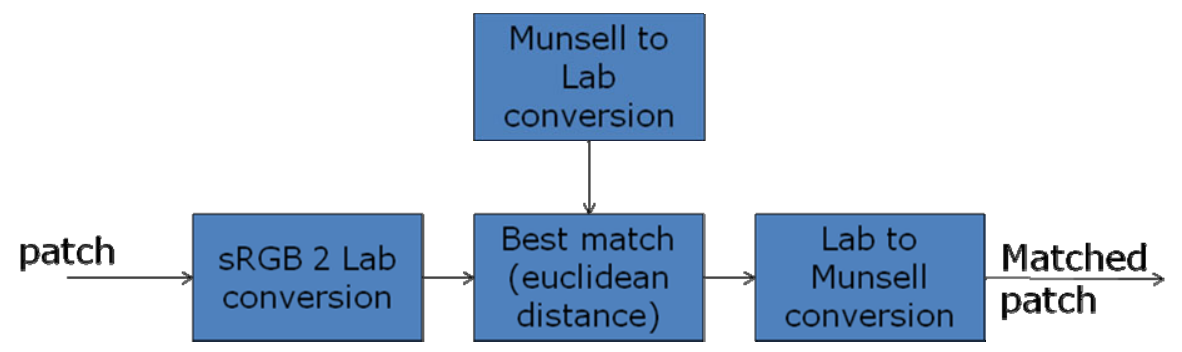

Fig. 7. Color matching block based scheme

Basically the patch is converted in the $L^{*} a^{*} b^{*}$ color space using the following:

$$
\begin{aligned}
& L^{*}=116 f\left(\frac{Y}{Y_{n}}\right)-16 \\
& a^{*}=500\left[f\left(\frac{X}{X_{n}}\right)-f\left(\frac{Y}{Y_{n}}\right)\right] \\
& b^{*}=200\left[f\left(\frac{Y}{Y_{n}}\right)-f\left(\frac{Z}{Z_{n}}\right)\right]
\end{aligned}
$$

All the patches of the Munsell table are also considered in the same color space. The best matching is performed using the minimum Euclidean distance between the patch and all the Munsell colors. The matched color is hence converted in the Munsell space and is provided to the user.

\section{Experimental Results}

First of all, we have tested that the proposed methods work correctly. To test this, we have acquired the image of the Munsell charts with the Macbeth color checker (Figure 8). 
In this way, the algorithm of color correction works well if the single patch has the correct color as shown in the Munsell table, in $90 \%$ of the experiments.

In order to test our proposed method, in Table 1 some results using the image of archaeological sherds [7] are presented. The second column shows the color suggested by the archaeologist. This attributed color is very subjective. The third column reports the more representative color in the sherd computed in the input image without any corrections. In the fourth and fifth columns the results of our techniques are shown.

Even if they are different from the human suggestion, they are very close to this. Hence, this means that the system works in the right direction.

We have observed that experimental results are close to the archaeologist suggestions with a success rate of $85 \%$ when the images are compensated instead of the original $73 \%$ for the uncorrected images.
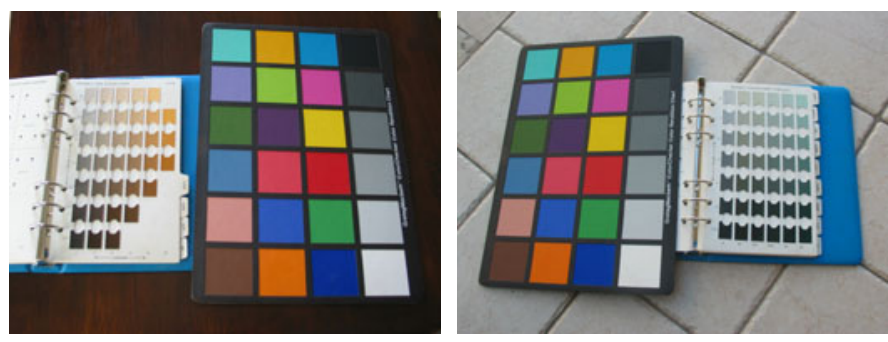

Fig. 8. Example of image used to validate the color matching block

Table 1. Some examples of color measures. The subjective archaeologist suggested color is compared with the algorithm results.

\begin{tabular}{|c|c|c|c|c|}
\hline Image & $\begin{array}{c}\text { Human } \\
\text { identification }\end{array}$ & $\begin{array}{c}\text { Without } \\
\text { correction }\end{array}$ & I method & II method \\
\hline 9570 & 7.5 YR 7/4 & 10 YR 6/4 & 7.5 YR 6/4 & 5 YR 7/4 \\
\hline 9579 & 10 R 7/6 & 5 YR 6/6 & 2.5 YR 7/4 & 5 YR 7/3 \\
\hline 9584 & 5 YR 6/2 & 10 YR 4/2 & 7.5 YR 6/2 & 5 YR 7/4 \\
\hline 9591 & 5 YR 6/1 & 7.5 YR 5/2 & 10 YR 5/1 & 7.5 YR 5/2 \\
\hline
\end{tabular}

\section{Conclusions}

A complete system to define the predominant color in archaeological sherds has been presented. It is an attempt to automate a manual methodology usually used by archeologists based on visual inspection and color matching of sherds (with Munsell table). Also a database has been created and it is available in [7].

The system aims to detect, starting from a single photo acquired with a common digital still camera, the real color of a patch pointed by the user and to retrieve the patch (in Munsell coordinates) with the best match. Color accuracy is important, but it cannot be ensured by the camera due to the critical illumination condition usually where the images are captured. The paper shows all the criticalities of the problem 
and proposes a methodology to overcome such problems. Particular attention has been used to select the proper color space and perceptive distance measures. Next steps will be focused on increase the reliability of the color correction, e.g., by using all the patches of the Macbeth chart or increasing the color accuracy for the patches nearer to the color of the find.

Next steps will be focused on increase the reliability of the color correction, e.g., by using a more perceptually uniform color space for the error measure shown in equation (4), or by using all the patches of the Macbeth chart or increasing the color accuracy for the patches nearer to the color of the find.

\section{References}

[1] Gerharz, R.R., Lantermann, R., Spennemann, D.R.: Munsell Color Charts: A Necessity for Archaeologists? Australian Historical Archaeology 6, 88-95 (1988)

[2] Goodwin, C.: Practices of Color Classification. Mind, Culture and Activity 7(1-2), 19-36 (2000)

[3] Conway, B.R., Livingstone, M.S.: A different point of hue. Proceedings of the National Academy of Sciences of the United States of America 102(31), 10761-10762 (2005)

[4] Blatner, D., Chavez, C., Fraser, B.: Real World Adobe Photoshop CS3. Peachpit press, Berkeley (2008)

[5] Munsell, A.H.: The Atlas of the Munsell Color System, Boston (1915)

[6] Bianco, S., Ciocca, G., Cusano, C., Schettini, R.: Improving Color Constancy Using Indoor-Outdoor Image Classification. IEEE Trans. on Image Processing 17(12) (2008)

[7] Database used in the experiments, http: //www.archeomatica.unict.it/sherds\&Macbeth.rar

[8] Hordely, S.D.: Scene illuminant estimation: Past, present, and future. Color Res. Appl. 31(4), 303-314 (2006)

[9] van de Weijer, J., Gevers, T., Gijsenij, A.: Edge-based Color Constancy. IEEE Trans. on Image Processing 16(9), 2207-2214 (2007)

[10] Gasparini, F., Schettini, R., Naccari, F., Bruna, A.R.: Multidomain pixel analysis for illuminant estimation. In: Proceedings of SPIE Electronic Imaging 2006 - Digital Photography II - 2006, San Josè (2006)

[11] Funt, B., Barnard, K., Martin, L.: Is machine colour constancy good enough? In: Burkhardt, H.-J., Neumann, B. (eds.) ECCV 1998. LNCS, vol. 1406, pp. 445-459. Springer, Heidelberg (1998)

[12] Von Kries, J.: Chromatic adaptation. Festchrift der Albrecht-Ludwigs-Universitat (1902) [Translation: MacAdam, D.L.: Colorimetry-Fundamentals. SPIE Milestone Series, vol. MS 77 (1993)]

[13] Nickerson, D.: The specification of color tolerance. Tex. Res. 6, 505-514 (1936) 